\title{
A Reliability Measure and Model Method of Space Station
}

\author{
XIA Qiaoli, a , HOU Yongqing ${ }^{2, b}$, WEI Chuanfeng ${ }^{3, c}$ \\ China Academy of Space Technology, Beijing 100094, China \\ aXiaqiaoli_buaa@163.com, 'houyongqing 11@hotmail.com, 'cdavidwei@126.com
}

Abstract: On-orbit maintenance is important means to sustain the reliability of space station. Reliability block diagram and static fault tree analysis are usually applied to measure the system reliability of space station. The results are too conservative due to the disregard of maintenance. Markov method can deal with the measure in case of maintenance. However, due to the possibility of state combinational explosion problem in Markov chain, it is difficult to apply in the space station. The paper investigates a reliability measurement method to consider the contribution of maintenance, and in this way the reliability can be calculated easily. First, check whether the reliability block diagram should be updated by the maintenance condition inspection, and convert the model into dynamic fault tree, then, use Markov transfer chain to calculate reliability. Examples are analyzed with these new methods. The analysis results show that the method can accurately measure the maintenance contribution to the reliability, and the analysis accuracy is better than RBD.

\section{Keywords: Space Station, Maintenance, Reliability, Dynamic Fault Tree 1 Introduction}

Space station is one kind of typical complex spacecraft need on-orbit maintenance, which life expectancy requires more than 10 years. A variety of failures may occur during the long-term orbital operation. These failures could affect the mission completion and even threaten the safety of the station. The research of foreign space station shows that, general space station long-life strategy includes three elements: firstly use the long-life design method to prolong product's service life; then strengthen the reliability and reduce the random fault; finally, supplement orbital maintenance to sustain high reliability. On-orbit maintenance is a kind of effective method to prevent faults and realize the life expectancy of space station. By means of maintenance, actual life of Mir space station extended to 15 years when its original design life is 5 years. The international space station uses RCM (reliability-centered maintenance) design so that its life has been more than 15 years.

The reliability measurement and modeling on the condition of maintainability design becomes a new problem. According to the reliability theory, system availability is the comprehensive reflection of reliability and maintainability. Availability represents the effectiveness of the system, that is, the ability of the system to work at any time [1]. If use availability as a design index during development phase of space station, the following problems are existed in the engineering application:

1. In order to reflect the integrity of the complex system, usually Markov process state method is applied to calculate availability [2]. The calculation of this method is complex, in addition, Markov model states should not be too much, otherwise, it will appear "state explosion” problem of the system modeling and calculation. Therefore, availability is not suitable as a design index. Research on ISS shows that availability is evaluated based on flight data, it also don't be used as a design index during the development stage.

2. Availability is related to maintenance support resources which cannot be assigned to subsystem and equipment. If assign availability to reliability and maintainability, maintainability is not suitable as a design index too. According to the definition of 
availability, availability index can also assign to MTBM (mean time between maintenance), RT (average waiting time) and MDT (administration, logistics and other reasons for the delay time). MTBM is still a complex parameter relates to reliability and maintainability design. Besides, we don’t have on-orbit maintenance time data, it is difficult to distribute and validate maintenance time.

As a result, it is not recommended to use availability as a design parameter during the development stage. Availability can be evaluated based on on-orbit flight data. During development stage, reliability under the condition of maintenance can be used as a design index.

Static reliability block diagram and fault tree model are usually used on spacecraft engineering to carry out the system reliability analysis and measurement, which cannot reflect the characteristics of on-orbit maintenance. Therefore, this paper investigates a reliability measurement method to consider the contribution of maintenance, combines with static reliability block diagram and dynamic fault tree to establish the reliability model of the system. The basic idea is: first establish static reliability block diagram, and then update RBD model if the units meet maintenance conditions, convert RBD to dynamic fault tree, then use Markov transfer probability formula to calculate dynamic fault tree[3]. System reliability can be obtained on the base of dynamic fault tree and RBD calculation result. Static reliability block diagram modeling and calculation method is simple and mature, which is not in this paper.

\section{Maintenance conditions validation}

Generally, mechanical products which has wear-out characteristics and key electronic equipment on space station will be identified to carry out the maintainability design [4]. Due to the product characteristics and on-orbit environment constraints, components like cabin structure, docking mechanism and radiator cannot be repaired, which need to apply reliability margin design and space environmental adaptability design to prolong service life. Including both repairable products and non-repairable products, both static reliability block diagram and dynamic reliability model should be used to model system reliability. Therefore, it is necessary to validate maintenance conditions of the component. Update the model if maintenance conditions are meet, otherwise, use reliability block diagram, so as to reduce the size of the dynamic model, reduce the amount of computation.

Maintenance condition validation start from equipment design plan, including the following steps:

1. First, validate the maintenance design of equipment from these aspects: fastener disassembly, electric connector plug, anti-leakage design, high precision installation ensure design, and operation space analysis etc. if all requirements are meet, then go to the next step, otherwise, use reliability block diagram method.

2. Then, validate the maintenance design of platform from these aspects: fault detection and isolation design, layout design to ensure accessibility, power isolation design, Information isolation design, after maintenance testability design etc. if all requirements are meet, then go to the next step, otherwise, use reliability block diagram method.

3. Finally, validate whether there are long-term on-orbit storage spare parts. If yes, update block diagram model to dynamic fault tree, if no, use reliability block diagram method.

The flow chart of maintenance condition inspection is shown in Fig. 1: 


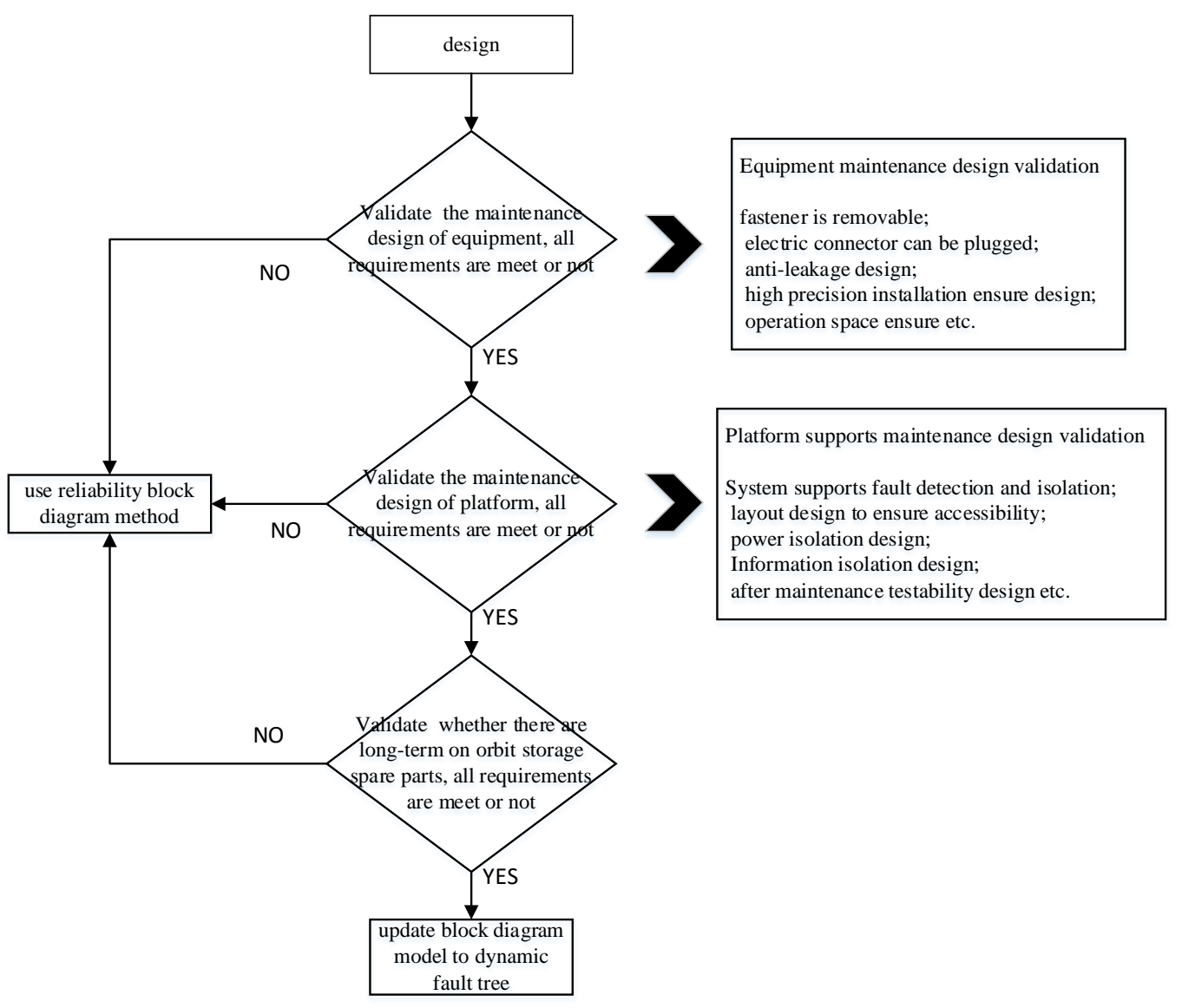

Fig.1 Maintenance conditions inspection process

\section{Reliability model update}

Taking the series model as an example, this paper introduces the model updating method of the common reliability block diagram of the space station system. The reliability of the series model is shown in Fig. 2:

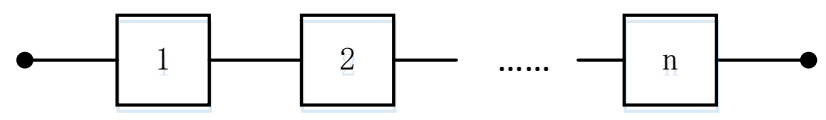

Fig.2 Reliability series model

For the sub system apply series model, assume following conditions are obeyed:

1. Unit $i, \quad i=1,2, \cdots n$ adopt universal design;

2. $\quad S 1$ is a long-term on orbit storage spare part, the spare part can be used to instead any failure unit.

3. Replacement is completed within the specified time, within which, the system still operate safely by adjusting working mode.

According to above three conditions, the on-orbit storage of spare parts $S 1$ is similar to a cold backup, switching time is equal to maintenance time, which is in the allowed scope. As the $S 1$ can be used as a cold standby to all units, and $S 1$ must be used after the failure of unit $i$. due to the resources sharing and the failure sequence correlation, conventional reliability block diagram cannot be able to describe system accurately. However, dynamic fault tree can be a very good description of the dynamic characteristics [5]. Therefore, using dynamic fault tree to modify series model like Fig. 3: 


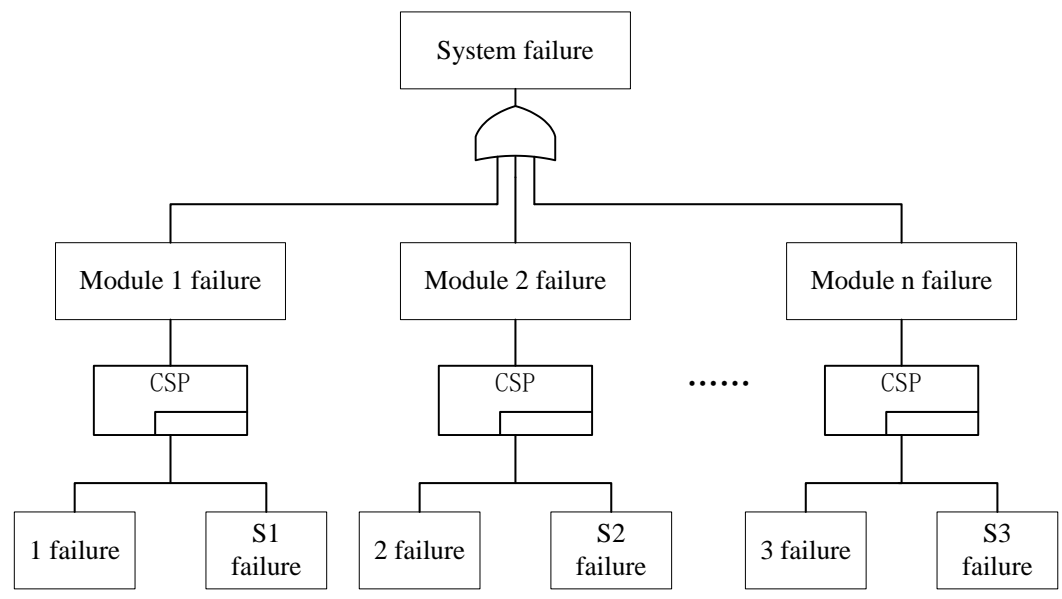

Fig.3 Dynamic fault tree

\section{Dynamic fault tree calculation}

Because of containing the dynamic logic gates, dynamic fault tree should use Markov process to calculate instead of traditional analysis method. However, the calculation of Markov process is complex because of using $\mathrm{L}$ transform to calculate differential equations. So, the method of probability calculation of transfer chain [3] is adopted in this paper which is simple and valuable to engineer application.

The probability calculation steps of the transfer chain are as follows:

Step 1: convert the dynamic fault tree to a Markov chain;

Step 2: divide the Markov chain into a number of state transition chain;

Step 3: calculate the probability of each chain according to the corresponding formula of different chain length;

Step 4: add the probability of the chains together, you can get the failure probability of the entire fault tree.

The calculation formula of failure probability of Markov chain, which length of the chain is $n$, is as shown in Eq. 1[3]:

$$
P_{n}(t)=\prod_{i=1}^{n} \lambda_{i-1, i}\left[\begin{array}{l}
\prod_{i=1}^{n} \frac{1}{\lambda_{i-1, i}+\lambda_{i-1, N F}}- \\
\sum_{i=1}^{n} \frac{e^{-\left(\lambda_{i-1, i}+\lambda_{i-1, N F}\right) t}}{\left(\lambda_{i-1, i}+\lambda_{i-1, N F}\right) \prod_{j=1, j \neq i}^{n}\left(-\lambda_{i-1, i}-\lambda_{i-1, N F}+\lambda_{j-1, j}+\lambda_{j-1, N F}\right)}
\end{array}\right]
$$

In Formula $1, \lambda_{0,1}>0 ; \lambda_{1,2}>0 ; \lambda_{1, N F} \geq 0$. “NF” Indicates the system is not in a state of failure.

The calculation procedure of the dynamic fault tree is introduced by taking some control function of the space station as an example. The control function consists of two units in series. One device is A, the other is B. A and B design the same. A spare part S is stored on orbit long-term, which can be used to replace either A or B when failure occurs. Establish the dynamic fault tree as shown in Fig. 4: 


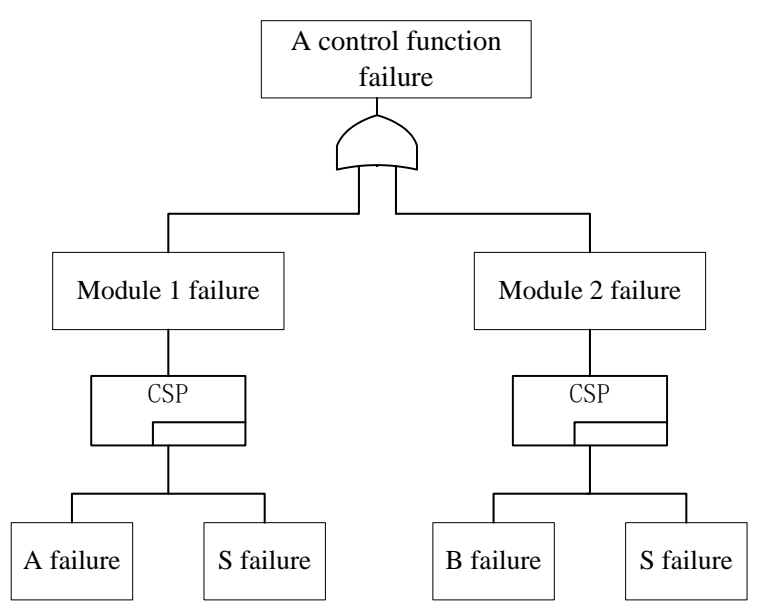

Fig.4 A control function failure dynamic fault tree

The "or" relationship between the two modules is static, which can be calculate by BDD method. The static layer BDD diagram of "some control function failure" is as shown in Fig. 5, Where $P 1$ represents "module 1", $P 2$ represents "module 2".

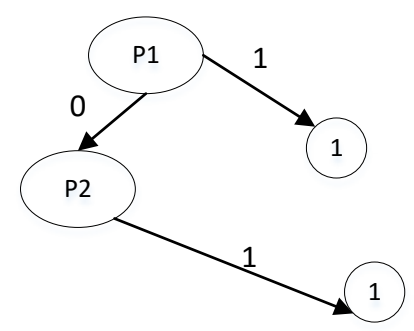

Fig.5 BDD decomposition process of a control function

Applying BDD method to calculate, the structure function is as shown in the Eq. 2:

$\Phi(P)=P_{1} \cup P_{2} \overline{P_{1}}=P_{1} \cup P_{2}$

When $\Phi(P)=1$, the system failure occur; when $\Phi(P)=0$, that the system failure did not occur. The probability of a system failure is calculated as shown in the Eq. 3:

$$
P\left(X_{P}\right)=P(\Phi(P)=1)=P\left(P_{1}\right)+P\left(P_{2}\right)
$$

The Cold Standby module 1 of the dynamic fault tree can be calculated by Markov chain. At $\mathrm{t}$ time, the possible states of system are as shown in the Eq. 4.

The state of the system at $t$ time is possible.

$$
X(t)=\left\{\begin{array}{l}
0, \quad \text { two devices are in good condition, and the system is normal } \\
1, \quad \text { A fault, and the system is normal } \\
F_{\mathrm{a}}, \quad \text { S fault after A failure, system failure }
\end{array}\right.
$$

The transfer process of cold standby is as shown in Fig. 6:

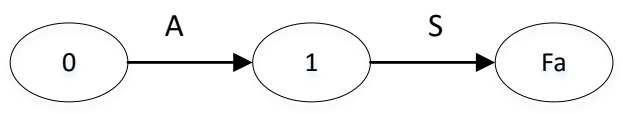

Fig.6 Markov chain of model 1

In the state transition diagram above, the state $F_{a}$ represents system failure. Back forward from the failure state, the transfer chain $\overrightarrow{A, S}$ can be gained. The probability of Markov chain length 
2, can be calculated by putting $\mathrm{N}=2$ into Formula 1 .

\section{An illustrative example}

The belief reliability evaluation of a control system is conducted in this section as an illustrative example, according to the DFT presented in Figure 4.

If $\lambda_{A}=6.3 \times 10^{-7} / h ; \lambda_{s}=6.5 \times 10^{-7} / h, t=26280 h$, the value of $P\left(P_{1}\right)$ and $P\left(P_{2}\right)$ can be calculated using formula 4 . The resulted $P\left(P_{1}\right)$ and $P\left(P_{2}\right)$ are $1.3983 \times 10^{-4}$ respectively.

The system failure probability $P\left(P_{X}\right)$ can be calculated using formula 3 . The reliability of system can be calculated using Eq. 5. The resulted $P\left(P_{X}\right)$ and $R(t)$ are $2.7967 \times 10^{-4}$ and 0.9997 , respectively.

$$
R(t)=1-P\left(X_{p}\right)=0.9997
$$

Calculate reliability block diagram as a comparison, the series model can be calculated using Eq. 6:

$$
R_{\text {series }}=\left(e^{-\lambda_{A} t}\right)^{2}
$$

The resulted $R_{\text {series }}$ is 0.9674 .

Through calculation and analysis, the result shows that Markov chain failure probability formula can be applied to calculate dynamic fault tree, which can get a better calculation than reliability block diagram method. It is in good agreement with the analysis that on-orbit maintenance can improve long -term mission reliability, which shows that the method in this paper can improve the accuracy of reliability measurement.

\section{Conclusion}

A system reliability modeling method of repairable spacecraft is investigated in this paper. Both static reliability block diagram and dynamic fault tree are applied in this method. Check whether the reliability block diagram should be updated by the maintenance condition inspection, then convert the model into dynamic fault tree, use Markov transfer chain to calculate reliability. Examples are analyzed with these new methods. The analysis results show that this method can solve the reliability measure and model problem of repairable spacecraft during development phase. At the same time, the method is simple and has a good engineering application prospect.

\section{References}

[1]Mario P B, Gwyn G. A Markov chain state transition approach to establishing critical phases for AUV reliability [J]. IEEE Journal of Oceanic Engineering, 2011, 36(1): 139-149.

[2]ZHANG Xiao-jie, ZHAO Hai-tao, Reliability Analysis of Satellite System Based on Dynamic Fault Tree [J]. Journal of Astronautics, 2009, 30(3): 1249-54.

[3]ZHANG Wei, XIA Qiaoli, Study on Design and Verification Method of Space Station Maintainability [J]. Manned Spaceflight, 2014, 20(2): 134-138.

[4]Leila Meshkat, J B Dugan, John D Andrews. Dependability analysis of system with on-demand and active failure models, using dynamic fault trees [J]. IEEE Trans. On Reliability, 2002, 51(2): 240-251.

[5]J B Dugan, David Coppit. Developing a low-cost high-quality software tool for dynamic fault-tree analysis [J]. IEEE Trans. On Reliability, 2000, 49(1): 49-59. 